\title{
Polymorphisms in regulators of xenobiotic transport and metabolism genes $P X R$ and $C A R$ do not affect multiple myeloma risk: a case-control study in the context of the IMMEnSE consortium
}

\author{
Alessandro Martino ${ }^{1}$, Juan Sainz ${ }^{2,3}$, Rui Manuel Reis ${ }^{4,5}$, Victor Moreno ${ }^{6}$, Gabriele Buda ${ }^{7}$, Fabienne Lesueur ${ }^{8}$, \\ Herlander Marques ${ }^{4}$, Ramón García-Sanz ${ }^{9}$, Rafael Ríos ${ }^{2,3}$, Angelika Stein ${ }^{1}$, Charles Dumontet ${ }^{10}$, \\ Federica Gemignani ${ }^{11}$, Anna Maria Rossi ${ }^{11}$, Stefano Landi ${ }^{11}$, Manuel Jurado ${ }^{2,3}$, Mario Petrini ${ }^{7}$, \\ Krzysztof Jamroziak $^{12}$, Daniele Campa ${ }^{1}$ and Federico Canzian ${ }^{1}$
}

The exposure to pesticides and toxic compounds in xenobiotic transport and metabolism genes has been shown to affect risk of developing multiple myeloma (MM). Therefore, we hypothesized that genetic variations in xenobiotic transport and metabolism regulator genes $P X R$ (NR1/2) and CAR (NR1/3) could determine a difference in MM susceptibility. Ten tagging singlenucleotide polymorphisms (SNPs) for PXR and seven for the CAR genes were selected and genotyped in 627 MM cases and 883 controls collected in the context of the International Multiple Myeloma rESEarch (IMMEnSE) consortium. None of the 17 SNPs investigated showed significant association with MM risk either alone or when combined in haplotypes. Significant SNPSNP interactions were not found, neither with 58 previously genotyped polymorphisms in ABC transporters. We can therefore exclude that common genetic variants in the xenobiotic transport and metabolism regulator genes PXR and CAR affect MM risk. Journal of Human Genetics (2013) 58, 155-159; doi:10.1038/jhg.2012.149; published online 10 January 2013

Keywords: association studies; CAR; genetic susceptibility; multiple myeloma; PXR

\section{INTRODUCTION}

Multiple myeloma (MM) is a hematological neoplasm with a worldwide incidence of 1.5/100 000 new cases/year. The disease is slightly more frequent in males than females, and the mean age at diagnosis is around 60 years. ${ }^{1}$ It has been shown that MM could evolve from an asymptomatic premalignant stage termed monoclonal gammopathy of undetermined significance (MGUS). The rate of progression of this condition to $\mathrm{MM}$ is around $1.5 \%$ per year, and about $15-17 \%$ of MGUS subjects develop MM. ${ }^{2}$ Among other proposed risk factors, pesticides and toxic compounds have been shown to affect the risk of developing MM and MGUS. ${ }^{3,4}$ In particular, higher incidence rates of both MM and MGUS have been shown among farmers and agricultural workers. ${ }^{3,5-7}$

In addition, converging evidences of MM in monozygotic twins and familial aggregation of MM strongly suggest that MM etiology has a robust genetic component. ${ }^{8}$ Several case-control studies investigating the role of single-nucleotide polymorphisms (SNPs) in MM risk have been conducted, and associations of SNPs in genes involved in xenobiotic metabolism with $\mathrm{MM}$ risk have been reported. ${ }^{9,10}$ Recently, we found a strong association of SNPs in the key xenobiotic transporter $A B C B 1$ gene with the risk of $\mathrm{MM}$ in the largest candidate-gene study on $\mathrm{MM}$ risk conducted to date. ${ }^{11}$ Both evidences that the exposure to toxic compounds, and in particular pesticides, can increase the risk of MM (and its premalignant condition MGUS) and that several SNPs within genes involved in xenobiotic transport and metabolism are associated with MM risk suggest that the xenobiotic metabolism process could have an important role in MM susceptibility and that genetic variation in key genes within this pathway can further affect the individual risk to develop MM. Although the role of genetic variation in xenobiotic

${ }^{1}$ Genomic Epidemiology Group, German Cancer Research Center DKFZ, Heidelberg, Germany; ${ }^{2}$ Genomic Oncology Area, Genyo-Pfizer-University of Granada-Andalusian Government Centre for Genomics and Oncological Research, Granada, Spain; ${ }^{3}$ Department of Hematology, Virgen de las Nieves University Hospital, Granada, Spain; ${ }^{4}$ Life and Health Sciences Research Institute (ICVS), School of Health Sciences, University of Minho, Braga, Portugal; ${ }^{5}$ Molecular Oncology Research Center, Barretos Cancer Hospital, Barretos, Brazil; ${ }^{6}$ Catalan Institute of Oncology, IDIBELL and CIBERESP, University of Barcelona, L'Hospitalet, Barcelona, Spain; ${ }^{7}$ Department of Oncology, Transplants and Advanced Technologies, Section of Hematology, Pisa University Hospital, Pisa, Italy; ${ }^{8}$ Genetic Cancer Susceptibility Group, International Agency for Research on Cancer (IARC), Lyon, France; ${ }^{9}$ University Hospital of Salamanca, Universidad de Salamanca-Consejo Superior de Investigaciones Científicas, Salamanca, Spain; ${ }^{10}$ INSERM UMR $1052 /$ CNRS 5286, Laboratoire de Cytologie Analytique, Faculté de Médecine Rockefeller, Université Claude Bernard Lyon I, Lyon, France; ${ }^{11}$ Department of Biology, Section of Genetics, University of Pisa, Pisa, Italy and ${ }^{12}$ Department of Hematology, Medical University of Lodz, Lodz, Poland

Correspondence: Dr F Canzian, Genomic Epidemiology Group, German Cancer Research Center (DKFZ), Im Neuenheimer Feld 280, D-69120 Heidelberg, Germany. E-mail: f.canzian@dkfz-heidelberg.de

Received 25 July 2012; revised 22 November 2012; accepted 29 November 2012; published online 10 January 2013 
metabolism enzymes and transporters has been already extensively studied, no study focused on the role of regulators of xenobiotic transport and metabolism.

The pregnane $\mathrm{X}$ receptor (PXR or NR1I2) and the constitutive androstane receptor (CAR or NR1I3) are the most known and wellcharacterized regulators of xenobiotic transport and metabolism. These proteins belong to the nuclear receptor subfamily and are activated by numerous xenobiotics, drugs, bile acids and hormones. It has been shown that several environmental, occupational and natural products, among which pesticides (some of which specifically linked with MM risk ${ }^{3,5-7}$ ), can activate (or inactivate) both PXR and CAR. ${ }^{12}$ The activation of PXR and CAR leads to the induction of several detoxification enzymes (that is, phase I cytochrome P450 enzymes, phase II enzymes, such as uridine diphospho-glucorunosyltransferase, glutathione-S-transferases and sulfutransferases, and the phase III transporters, such as ABCB1 and ABCG2). ${ }^{12}$ In addition, PXR and $C A R$ have shown to be highly polymorphic genes with several SNPs identified within their coding or regulatory sequences. Indeed, genetic variants within $P X R$ and $C A R$ genes have been related to differential gene expression of some of the detoxifying enzymes activated by these receptors. ${ }^{12-14}$ For this reason, it has been hypothesized that part of the individual variability in xenobiotic metabolism efficiency could be due to the genetic variations within these regulator genes. ${ }^{15}$

Therefore, to address the hypothesis that genetic variants in PXR and $C A R$ genes could affect the risk of MM, we selected 17 SNPs in the PXR and CAR genes and genotyped them in $627 \mathrm{MM}$ cases and 883 controls.

\section{MATERIALS AND METHODS}

\section{Study population}

The study population consisted of $627 \mathrm{MM}$ cases and 883 controls (Table 1). This study way carried out in the context of the International Multiple Myeloma rESEarch (IMMEnSE) consortium, a recently created working group that aims to study genetics and pharmacogenetics of MM. ${ }^{10,16,17}$ Briefly, cases were defined by a confirmed diagnosis of $\mathrm{MM}$, according to the International Myeloma Working Group criteria. ${ }^{18}$ Incident cases of MM diagnosed between 1990-2010 were recruited, whereas subjects with uncertain diagnosis or MGUS were excluded. Different region-specific subpopulations of controls were selected among the general population, as well as among hospitalized subjects with different diagnoses, excluding cancer.

For each subject, informed consent to collect fresh blood and perform DNA extraction for research purpose was requested and collected individually by each center. The study has been approved by the competent ethical committees.

\section{SNP selection and genotyping}

We used a tagging SNP approach for the selection of the genetic variants within the PXR and the CAR genes. The entire set of common genetic variants (including $5 \mathrm{~kb}$ upstream of the first exon and $5 \mathrm{~kb}$ downstream of the last exon of each gene), with minor allele frequency (MAF) $\geqslant 5 \%$ in Caucasians from the International HapMap Project (release no. 26; http://www.hapmap. org), was included for both genes. Tagging SNPs were selected using the Tagger algorithm available through Haploview (http://www.broad.mit.edu/mpg/ haploview/; http://www.broad.mit.edu/mpg/tagger/), using pairwise SNP selection with a minimum $r^{2}$ threshold of 0.8 . This selection resulted in 10 tagging SNPs for PXR and 7 for CAR. Genotyping was carried out at the German Cancer Research Center (DKFZ) in Heidelberg, where the IMMEnSE bio-bank is set up, on genomic DNA extracted from peripheral blood, using both TaqMan (Applied Biosystems, Foster City, CA, USA) and KASPar (KBioscence, Hoddesdon, UK) technologies. The order of DNAs from cases and controls was randomized on plates to ensure that an equal number of cases and controls were analyzed simultaneously. For quality-control purpose, duplicates of $10 \%$ of the samples were interspersed throughout the plates. PCR plates for both TaqMan and KASPar assays were read on an ABI PRISM 7900HT instrument (Applied Biosystems). The SDS Software, version 2.4 (Applied Biosystems) was used to determine the genotypes. Subjects whose samples had a call rate $<75 \%$ were dropped from any analysis. Once subjects with low call rate were removed, all SNPs had a call rate $>95 \%$.

\section{Statistical analysis}

The Hardy-Weinberg equilibrium was tested in controls for each polymorphism. The $\chi^{2}$ - and Kruskal-Wallis tests were used to compare gender and age distributions between MM patients and controls. Unconditional logistic regression was used to assess the main effects of the genetic polymorphisms on MM risk using a co-dominant and a dominant inheritance model. For each SNP, the more common allele in the controls was assigned as the reference category. All analyses were adjusted for age (continuous), gender and region of origin, and were conducted with STATA software (StataCorp, College Station, TX, USA).

Because of the large number of SNPs analyzed, a conservative threshold for statistical significance was applied, based on a revised version of the Bonferroni method. ${ }^{19}$

Haplotype blocks were identified from the genotyping data of the controls using SNPtool (http://www.dkfz.de/de/molgen_epidemiology/tools/ SNPtool.html ${ }^{20}$ and the Haploview v4.2 software. A MAF $>0.05$, HardyWeinberg equilibrium $P>0.001$ and a call rate $>75 \%$ were used as cut-off values. Individual haplotypes were then statistically inferred using the PHASE v.2.1.1 algorithm, based on a Bayesian approach (http://www.stat.Washington. edu/stephens/). ${ }^{21,22}$ Unconditional logistic regression was used to estimate risk. The most frequent haplotype was set as reference, whereas haplotypes with a frequency $<3 \%$ were declared as rare and combined in a single category.

Table 1 Demographical characteristics of IMMEnSE cases and controls used in this study

\begin{tabular}{|c|c|c|c|c|c|c|c|}
\hline \multirow[b]{2}{*}{ Region ${ }^{\mathrm{a}}$} & \multicolumn{3}{|c|}{ Cases } & \multicolumn{3}{|c|}{ Controls } & \multirow[b]{2}{*}{ Control type } \\
\hline & Gender M/F (Tot) & Mean Age ( \pm S.d.) & Median Age (Range) & Gender M/F (Tot) & Mean Age ( \pm S.d.) & Median Age (Range) & \\
\hline IT & 114/107 (221) & $62.7( \pm 9.8)$ & $63(35-87)$ & 124/104 (228) & $58.9( \pm 10.9)$ & $59(35-89)$ & General population \\
\hline PL & 49/48 (97) & $62.1( \pm 10.4)$ & $63(39-86)$ & $67 / 78(145)$ & $69.5( \pm 6.7)$ & $69(55-98)$ & Blood donors \\
\hline ES & 84/89 (173) & $62.8( \pm 11.4)$ & $62(31-93)$ & 168/151 (319) & $65.2( \pm 12.6)$ & $66(24-92)$ & Hospitalized subjects \\
\hline FR & 44/33 (77) & $55.9( \pm 9.0)$ & $57(34-75)$ & 46/46 (92) & $33.0( \pm 14.8)$ & $30(18-60)$ & Blood donors \\
\hline PT & 29/30 (59) & $67.2( \pm 10.3)$ & $69(43-86)$ & $54 / 45$ (99) & $60.7( \pm 7.7)$ & $58(51-85)$ & Blood donors \\
\hline Total & $320 / 307$ (627) & $62.2( \pm 10.6)$ & 62 (31-93) & 459/424 (883) & $60.4( \pm 15.0)$ & 62 (18-98) & \\
\hline
\end{tabular}

Abbreviations: ES, Spain; FR, France; IMMEnSE, International Multiple Myeloma rESEarch; IT, Italy; PL, Poland; PT, Portugal.

aIT: Department of Oncology, Transplants and Advanced Technologies, Section of Hematology, Pisa University Hospital, PISA; Department of Biology, Division of Genetics, Pisa University, PISA. PL: Department of Hematology, Medical University of Lodz, LODZ. ES: Division of Hematology, University Hospital of Salamanca, SALAMANCA; Hematology and Hemotherapy Department, University Department of Hematology, Medical University of Lodz, LODZ. ES: Division of Hematology, University Hospital of Salamanca, SALAMANCA; Hematology and Hemotherapy Department, University
Hospital Virgen de las Nieves, GRANADA; IDIBELL-Catalan Institute of Oncology, CIBERESP and Barcelona University, BARCELONA. FR: Hospices Civils de Lyon, LYON; International Agency for Research on Cancer IARC, LYON. PT: Life and Health Sciences Research Institute (ICVS), School of Health Sciences, University of Minho, BRAGA. 
Table 2 Genotype distribution of the typed SNPs in PXR and CAR genes among MM cases and controls

\begin{tabular}{llllll}
\hline SNP (rs) Cases (\%) & Controls (\%) & OR & $95 \% \mathrm{Cl}$ & P-value & P-trend
\end{tabular}

\begin{tabular}{lccccc}
\multicolumn{2}{l}{ PXR rs 10511395} & & & & \\
C/C & $429(69.5)$ & $623(70.7)$ & 1.00 & Ref & \\
A/C & $160(25.9)$ & $228(25.9)$ & 1.02 & $0.81-1.30$ & 0.85 \\
A/A & $28(4.6)$ & $30(3.4)$ & 1.38 & $0.81-2.35$ & 0.232 \\
A/C + A/A & $188(30.5)$ & $258(29.3)$ & 1.06 & $0.85-1.33$ & 0.588
\end{tabular}

PXR rs 1054190

$\begin{array}{lccccc}\mathrm{C} / \mathrm{C} & 452(74.0) & 656(74.8) & 1.00 & \text { Ref } & \\ \mathrm{C} / \mathrm{T} & 137(22.4) & 200(22.8) & 1.00 & 0.78-1.28 & 0.993 \\ \mathrm{~T} / \mathrm{T} & 22(3.6) & 21(2.4) & 1.56 & 0.84-2.88 & 0.155 \\ \mathrm{C} / \mathrm{T}+\mathrm{T} / \mathrm{T} & 159(26.0) & 221(25.2) & 1.05 & 0.83-1.34 & 0.680\end{array}$

PXR rs11917714

$\begin{array}{lccccc}\mathrm{C} / \mathrm{C} & 412(65.9) & 591(67.2) & 1.00 & \text { Ref } & \\ \mathrm{C} / \mathrm{T} & 190(30.4) & 250(28.5) & 1.07 & 0.85-1.35 & 0.535 \\ \mathrm{~T} / \mathrm{T} & 23(3.7) & 38(4.3) & 0.84 & 0.49-1.44 & 0.536 \\ \mathrm{C} / \mathrm{T}+\mathrm{T} / \mathrm{T} & 213(34.1) & 288(32.8) & 1.04 & 0.84-1.30 & 0.698\end{array}$

PXR rs 12488820

$\begin{array}{llllcl}\mathrm{C} / \mathrm{C} & 223(36.3) & 296(33.7) & 1.00 & \text { Ref } & \\ \mathrm{C} / \mathrm{T} & 289(47.0) & 407(46.4) & 0.93 & 0.74-1.18 & 0.574 \\ \mathrm{~T} / \mathrm{T} & 103(16.7) & 175(19.9) & 0.79 & 0.58-1.06 & 0.119 \\ \mathrm{C} / \mathrm{T}+\mathrm{T} / \mathrm{T} & 392(63.7) & 562(66.3) & 0.89 & 0.72-1.11 & 0.299\end{array}$

PXR rs 13071341

$\begin{array}{lccccc}\text { G/G } & 430(69.6) & 593(67.4) & 1.00 & \text { Ref } & \\ A / G & 166(26.9) & 269(30.6) & 0.85 & 0.67-1.07 & 0.158 \\ A / A & 22(3.5) & 18(2.0) & 1.70 & 0.90-3.22 & 0.102 \\ A / G+A / A & 188(30.4) & 287(32.6) & 0.90 & 0.72-1.12 & 0.355\end{array}$

\section{PXR rs3732359}

$\begin{array}{lccccc}A / A & 352(58.7) & 516(39.4) & 1.00 & \text { Ref } & \\ A / G & 209(34.8) & 291(33.5) & 1.04 & 0.83-1.30 & 0.720 \\ \text { G/G } & 39(6.5) & 62(7.1) & 0.90 & 0.59-1.37 & 0.619 \\ A / G+G / G & 248(41.3) & 353(40.6) & 1.02 & 0.82-1.26 & 0.881\end{array}$

\section{PXR rs13059232}

$\begin{array}{lrrrcc}\mathrm{C} / \mathrm{C} & 255(41.2) & 383(43.6) & 1.00 & \text { Ref } & \\ \mathrm{C} / \mathrm{T} & 299(48.3) & 390(44.4) & 1.16 & 0.93-1.44 & 0.192 \\ \mathrm{~T} / \mathrm{T} & 65(10.5) & 106(12.0) & 0.94 & 0.66-1.33 & 0.711 \\ \mathrm{C} / \mathrm{T}+\mathrm{T} / \mathrm{T} & 364(58.8) & 496(56.4) & 1.11 & 0.90-1.37 & 0.326\end{array}$

\section{PXR rs3732357}

$\begin{array}{lrcccc}\text { A/A } & 300(48.7) & 437(49.7) & 1.00 & \text { Ref } & \\ \text { A/G } & 240(39.0) & 361(41.0) & 0.94 & 0.75-1.17 & 0.589 \\ \text { G/G } & 76(12.3) & 82(9.3) & 1.31 & 0.92-1.85 & 0.130 \\ \text { A/G+ G/G } & 316(51.3) & 443(50.3) & 1.01 & 0.82-1.24 & 0.934\end{array}$

\section{PXR rs 1357459}

$\begin{array}{lccccc}\mathrm{T} / \mathrm{T} & 328(53.6) & 463(52.9) & 1.00 & \text { Ref } & \\ \mathrm{C} / \mathrm{T} & 249(40.7) & 345(39.4) & 1.02 & 0.82-1.27 & 0.850 \\ \mathrm{C} / \mathrm{C} & 35(5.7) & 67(7.7) & 0.75 & 0.49-1.17 & 0.206 \\ \mathrm{C} / \mathrm{T}+\mathrm{C} / \mathrm{C} & 274(46.4) & 412(47.1) & 0.98 & 0.79-1.20 & 0.838\end{array}$

\section{CAR rs3003596}

$\begin{array}{llllcl}\text { A/A } & 218(35.4) & 335(38.1) & 1.00 & \text { Ref } & \\ \text { A/G } & 296(48.0) & 393(44.7) & 1.16 & 0.93-1.46 & 0.191 \\ \text { G/G } & 102(16.6) & 151(17.2) & 1.04 & 0.77-1.41 & 0.799 \\ \text { A/G + G/G } & 398(64.6) & 544(61.9) & 1.13 & 0.91-1.40 & 0.264\end{array}$

0.981 0.815
Table 2 (Continued)

\begin{tabular}{|c|c|c|c|c|c|c|}
\hline$S N P(r s)$ & Cases (\%) & Controls (\%) & $O R^{\mathrm{a}}$ & $95 \% \mathrm{Cl}$ & $\mathrm{P}$-value & P-trend \\
\hline \multicolumn{7}{|c|}{ CAR rs3813627 } \\
\hline $\mathrm{G} / \mathrm{G}$ & $264(42.7)$ & $371(42.0)$ & 1.00 & Ref & & \multirow[t]{4}{*}{0.642} \\
\hline $\mathrm{G} / \mathrm{T}$ & $276(44.7)$ & $392(44.4)$ & 0.98 & $0.79-1.23$ & 0.882 & \\
\hline $\mathrm{T} / \mathrm{T}$ & $78(12.6)$ & $120(13.6)$ & 0.91 & $0.66-1.26$ & 0.581 & \\
\hline $\mathrm{G} / \mathrm{T}+\mathrm{T} / \mathrm{T}$ & $354(57.3)$ & $512(58.0)$ & 0.97 & $0.78-1.19$ & 0.750 & \\
\hline \multicolumn{7}{|c|}{ CAR rs 11265571} \\
\hline $\mathrm{A} / \mathrm{A}$ & $441(73.1)$ & $635(73.5)$ & 1.00 & Ref & & \multirow[t]{4}{*}{0.911} \\
\hline $\mathrm{A} / \mathrm{T}$ & $147(24.4)$ & $207(24.0)$ & 1.01 & $0.79-1.29$ & 0.911 & \\
\hline $\mathrm{T} / \mathrm{T}$ & $15(2.5)$ & $22(2.5)$ & 0.97 & $0.49-1.89$ & 0.921 & \\
\hline $\mathrm{A} / \mathrm{T}+\mathrm{T} / \mathrm{T}$ & $162(26.9)$ & $229(26.5)$ & 1.01 & $0.80-1.28$ & 0.937 & \\
\hline \multicolumn{7}{|c|}{ CAR rs2307418 } \\
\hline $\mathrm{T} / \mathrm{T}$ & $404(64.2)$ & $575(65.7)$ & 1.00 & Ref & & \multirow[t]{4}{*}{0.836} \\
\hline $\mathrm{G} / \mathrm{T}$ & $193(31.1)$ & $268(30.6)$ & 1.02 & $0.81-1.27$ & 0.879 & \\
\hline $\mathrm{G} / \mathrm{G}$ & $23(3.7)$ & $32(3.7)$ & 1.05 & $0.60-1.83$ & 0.863 & \\
\hline $\mathrm{G} / \mathrm{T}+\mathrm{G} / \mathrm{G}$ & $216(34.8)$ & $300(34.3)$ & 1.02 & $0.82-1.27$ & 0.851 & \\
\hline \multicolumn{7}{|c|}{ CAR rs2502805 } \\
\hline $\mathrm{C} / \mathrm{C}$ & $348(56.6)$ & $508(57.7)$ & 1.00 & Ref & & \multirow[t]{4}{*}{0.527} \\
\hline $\mathrm{C} / \mathrm{T}$ & $220(35.8)$ & $313(35.6)$ & 1.05 & $0.84-1.30$ & 0.693 & \\
\hline $\mathrm{T} / \mathrm{T}$ & $47(7.6)$ & $59(6.7)$ & 1.16 & $0.77-1.74$ & 0.484 & \\
\hline $\mathrm{C} / \mathrm{T}+\mathrm{T} / \mathrm{T}$ & $267(43.4)$ & $372(42.3)$ & 1.06 & $0.86-1.31$ & 0.564 & \\
\hline
\end{tabular}

\begin{tabular}{lrllcl}
\multicolumn{1}{l}{ CAR rs4073054 } & & \multicolumn{4}{l}{} \\
A/A & $245(39.8)$ & $346(39.4)$ & 1.00 & Ref & \\
A/C & $291(47.2)$ & $412(46.9)$ & 0.98 & $0.78-1.22$ & 0.855 \\
C/C & $80(13.0)$ & $120(13.7)$ & 0.94 & $0.68-1.31$ & 0.720 \\
A/C + C/C & $371(60.2)$ & $532(60.6)$ & 0.97 & $0.78-1.20$ & 0.785
\end{tabular}

\section{CAR rs4233368}

$\begin{array}{lccccc}C / C & 360(57.6) & 524(59.8) & 1.00 & \text { Ref } & \\ A / C & 225(36.0) & 302(34.4) & 1.09 & 0.88-1.36 & 0.439 \\ A / A & 40(6.4) & 51(5.8) & 1.15 & 0.74-1.78 & 0.538 \\ A / C+A / A & 265(42.4) & 353(40.2) & 1.10 & 0.89-1.35 & 0.377\end{array}$

Abbreviations: $\mathrm{Cl}$, confidence interval; $\mathrm{MM}$, multiple myeloma; OR, odds ratio; SNP, singlenucleotide polymorphism.

${ }^{a}$ ORs are adjusted for age, gender and region of recruitment. Differences in sample numbers are due to failures in genotyping.

SNP-SNP interactions were tested using the nonparametric Multifactor Dimensionality Reduction approach. Detailed information is described elsewhere, ${ }^{23}$ and the Multifactor Dimensionality Reduction software is available from http://www.epistasis.org.

\section{RESULTS AND DISCUSSION}

MM cases and controls had similar gender and age distributions $0.424\left(\chi^{2}=0.717\right.$, Kruskal-Wallis $\left.=0.39\right)$. All the SNPs were in HardyWeinberg equilibrium among controls $(P>0.02)$, with the exception of the PXR rs2461818 $(P=0.0003)$, which was therefore excluded from further analysis.

To correct for multiple testing, we calculated $M_{\text {eff }}$ values for each candidate gene separately $(\mathrm{PXR}=8, \mathrm{CAR}=3)$ and for the whole study (by adding the individual $M_{\text {eff }}$ values). The study-wise $M_{\text {eff }}$ was 11. We therefore used a study-wise statistical significance $P$-value of $0.05 / 11=0.0045$. None of the SNPs resulted associated with MM risk, with either a co-dominant or dominant inheritance model (Table 2). 
Table 3 Haplotype blocks in PXR and CAR genes and their distribution among MM cases and controls in the IMMEnSE population

\begin{tabular}{|c|c|c|c|c|c|c|c|}
\hline Gene & SNPs in haplotype block & Haplotypes & Cases N (\%) & Controls N (\%) & $O R^{a}$ & $\mathrm{Cl}$ & P-value \\
\hline \multirow[t]{4}{*}{ PXR Block 1} & rs12488820, rs13071341 & CG & $741(59.1)$ & $1004(56.9)$ & 1.00 & Ref & \\
\hline & & TG & $301(24.0)$ & $456(25.8)$ & 0.90 & $0.75-1.07$ & 0.225 \\
\hline & & $\mathrm{TA}$ & $207(16.5)$ & $306(17.3)$ & 0.92 & $0.75-1.12$ & 0.396 \\
\hline & & $\mathrm{CA}$ & $5(0.4)$ & - & & & \\
\hline \multirow[t]{5}{*}{ PXR Block 2} & rs11917714, rs3732359, & CACC & $720(57.4)$ & $1050(59.5)$ & 1.00 & Ref & \\
\hline & rs10511395, rs1054190 & TGCC & $235(18.7)$ & $330(18.7)$ & 1.02 & $0.84-1.24$ & 0.822 \\
\hline & & CAAT & $181(14.4)$ & $241(13.6)$ & 1.10 & $0.89-1.36$ & 0.385 \\
\hline & & CGCC & $66(5.3)$ & $93(5.3)$ & 1.02 & $0.73-1.42$ & 0.914 \\
\hline & & Others & $52(4.2)$ & $52(2.9)$ & 1.43 & $0.96-2.13$ & 0.076 \\
\hline \multirow[t]{6}{*}{ CAR Block 1} & rs4233368, rs4073054, & CCTACA & $427(34.0)$ & $630(35.7)$ & 1.00 & Ref & \\
\hline & rs2307418, rs3003596, & AATGTA & $286(22.8)$ & $388(22.0)$ & 1.09 & $0.90-1.33$ & 0.378 \\
\hline & rs2502805 rs11265571 & CAGACA & $235(18.7)$ & $331(18.7)$ & 1.05 & $0.85-1.30$ & 0.628 \\
\hline & & CATGCT & $165(13.2)$ & $250(14.2)$ & 0.97 & $0.77-1.23$ & 0.830 \\
\hline & & CATACA & $56(4.5)$ & $82(4.6)$ & 1.00 & $0.70-1.43$ & 0.990 \\
\hline & & Others & $85(6.8)$ & 85 (4.8) & 1.51 & $1.09-2.10$ & 0.014 \\
\hline
\end{tabular}

Abbreviations: IMMEnSE, International Multiple Myeloma rESEarch; MM, multiple myeloma; OR, odds ratio; SNP, single-nucleotide polymorphism.

Haplotype distribution among MM cases and controls for PXR and CAR genes in the IMMEnSE population.

a ORs are adjusted for age, sex and region of origin. Results in bold show $P<0.05$.

We reconstructed haplotypes within PXR and CAR genes based on the linkage disequilibrium blocks observed in the controls. Two haplotype blocks were identified in the PXR and one in the CAR gene. None of the individual haplotypes showed association with MM risk (Table 3). The statistically significant association for the others group (that is, rare haplotypes lumped together) within the CAR haplotype block is most likely because of the heterogeneity of the group. Further analyses within this group are prevented by the small numbers of each individual haplotype.

Finally, we evaluated SNP-SNP interactions using the nonparametric Multifactor Dimensionality Reduction approach. We investigated the interaction between PXR and CAR genotypes. In addition, we verified the presence of inter-gene interactions between the $P X R$ and $C A R$ genotypes and 58 previously genotyped polymorphisms in ABC transporters $A B C B 1, A B C G 2, A B C C 2, A B C C 1$ and $A B C C 3 .{ }^{11}$ We performed a stratified analysis of $P X R$ and $C A R$ genotypes among groups defined according to $A B C B 1$ genotypes that we previously showed to be strongly associated with $\mathrm{MM}$ risk. ${ }^{11}$ Nevertheless, no evidence of associations with $\mathrm{MM}$ risk emerged in any case (Supplementary Table I).

At the best of our knowledge, this is the first investigation of the $P X R$ and CAR genetic variation in relation to MM risk. Despite the strong rationale and the existing evidences suggesting a role of genetic variants in xenobiotic transport and metabolism genes in influencing MM risk, our results show no effect of SNPs in PXR and CAR genes in modifying individual susceptibility to the disease. In this study, we analyzed also the haplotype structure within the two genes and the possible SNP-SNP interactions, including the analysis of previously obtained genotypes for SNPs within ABC transporters. Moreover, our study had a sufficient statistical power to evidence the effect of common low-penetrance genetic variants, such as those expected to affect cancer susceptibility. Indeed, we achieved a statistical power of $80 \%$ to discover a minimum odds ratio (OR) of 1.34 with the smallest MAF observed among the selected tagging SNPs (0.14, rs1054190) and $\alpha=0.05$. The minimum OR detectable with the same power decreased to 1.24 with the highest MAF observed (0.43, rs12488820). Thus, we had sufficient power to detect risks of the magnitude commonly observed with genetic polymorphisms.
In the light of our thorough investigation showing overall negative results, we are keen to exclude a role of common genetic variations in the xenobiotic transporter and metabolism regulator genes $P X R$ and CAR in MM etiology. Although the tag SNP approach accounts for $>95 \%$ of the genetic variations with a MAF $\geqslant 0.05$ in both genes, including functional variants, we cannot exclude the presence of functional SNPs with a lower frequency that could still affect MM risk. Further studies in this sense are needed to identify and establish genetic risk factors for MM.

\section{CONFLICT OF INTEREST}

The authors declare no conflict of interest.

\section{ACKNOWLEDGEMENTS}

We thank for the support by the recruiting hospitals and physicians of the study regions, as well as their collaborating nurses and technicians.

Author contributions: AM, DC and FC designed the study, performed the genotyping and the statistical analysis and wrote the original draft of the manuscript. AS performed the genotyping. JS, RMR, VM, GB, FL, HM, RG-S, RR, CD, FG, AMR, SL, MJ, MP and KJ recruited cases and controls and provided DNA samples and the clinical information. All the authors approved the final version submitted.

1 Ferlay, J., Shin, H. R., Bray, F., Forman, D., Mathers, C. \& Parkin, D. M. GLOBocAN 2008 v2.0, Cancer Incidence and Mortality Worldwide: IARC CancerBase No. 10 [Internet] (International Agency for Research on Cancer, Lyon, France, 2010, Available from: http://globocan.iarc.fr).

2 Kyle, R. A., Therneau, T. M., Rajkumar, S. V., Larson, D. R., Plevak, M. F. \& Melton, 3rd L. J. Long-term follow-up of 241 patients with monoclonal gammopathy of undetermined significance: the original Mayo Clinic series 25 years later. Mayo Clin. Proc. 79, 859-866 (2004).

3 Landgren, O., Kyle, R. A., Hoppin, J. A., Beane Freeman, L. E., Cerhan, J. R., Katzmann, J. A. et al. Pesticide exposure and risk of monoclonal gammopathy of undetermined significance in the Agricultural Health Study. Blood 113, 6386-6391 (2009).

4 Lope, V., Perez-Gomez, B., Aragones, N., Lopez-Abente, G., Gustavsson, P., Plato, N. et al. Occupation, exposure to chemicals, sensitizing agents, and risk of multiple myeloma in Sweden. Cancer Epidemiol. Biomarkers Prev. 17, 3123-3127 (2008).

5 Koutros, S., Alavanja, M. C., Lubin, J. H., Sandler, D. P., Hoppin, J. A., Lynch, C. F. et al. An update of cancer incidence in the Agricultural Health Study. J. Occup. Environ. Med. 52, 1098-1105 (2010). 
6 Perrotta, C., Staines, A. \& Cocco, P. Multiple myeloma and farming. A systematic review of 30 years of research. Where next? J. Occup. Med. Toxicol. 3, 27 (2008).

7 Pahwa, P., Karunanayake, C. P., Dosman, J. A., Spinelli, J. J., McDuffie, H. H. \& McLaughlin, J. R. Multiple myeloma and exposure to pesticides: a Canadian casecontrol study. J. Agromed. 17, 40-50 (2011).

8 Landgren, O. \& Weiss, B. M. Patterns of monoclonal gammopathy of undetermined significance and multiple myeloma in various ethnic/racial groups: support for genetic factors in pathogenesis. Leukemia 23, 1691-1697 (2009).

9 Lincz, L. F., Kerridge, I., Scorgie, F. E., Bailey, M., Enno, A. \& Spencer, A. Xenobiotic gene polymorphisms and susceptibility to multiple myeloma. Haematologica 89, 628-629 (2004).

10 Martino, A., Sainz, J., Buda, G., Jamroziak, K., Reis, R. M., Garcia-Sanz, R. et al. Genetics and molecular epidemiology of multiple myeloma: the rationale for the IMMEnSE consortium (review). Int. J. Oncol. 40, 625-638 (2011).

11 Martino, A., Campa, D., Buda, G., Sainz, J., Garcia-Sanz, R., Jamroziak, K. et al. Polymorphisms in xenobiotic transporters ABCB1, ABCG2, ABCC2, ABCC1, ABCC3 and multiple myeloma risk: a case-control study in the context of the International Multiple Myeloma rESEarch (IMMEnSE) consortium. Leukemia 26, 1419-1422 (2011).

12 Hernandez, J. P., Mota, L. C. \& Baldwin, W. S. Activation of CAR and PXR by dietary, environmental and occupational chemicals alters drug metabolism, intermediary metabolism, and cell proliferation. Curr. Pharmacogenomics Person. Med. 7, 81-105 (2009).

13 Zhang, B., Xie, W. \& Krasowski, M. D. PXR: a xenobiotic receptor of diverse function implicated in pharmacogenetics. Pharmacogenomics 9, 1695-1709 (2008).
14 Lamba, J. K. Pharmacogenetics of the constitutive androstane receptor. Pharmacogenomics 9, 71-83 (2008)

15 Timsit, Y. E. \& Negisi, M. CAR and PXR: the xenobiotic-sensing receptors. Steroids 72 , 231-246 (2007).

16 Campa, D., Martino, A., Sainz, J., Buda, G., Jamroziak, K., Weinhold, N. et al. Comprehensive investigation of genetic variation in the $8 \mathrm{q} 24$ region and multiple myeloma risk in the IMMEnSE consortium. Br. J. Haematol. 157, 331-338 (2012).

17 Martino, A., Campa, D., Jamroziak, K., Reis, R. M., Sainz, J., Buda, G. et al. Impact of polymorphic variation at $7 \mathrm{p} 15.3,3 \mathrm{p} 22.1$ and $2 \mathrm{p} 23.3$ loci on risk of multiple myeloma. Br. J. Haematol. 158, 805-809 (2012).

18 International Myeloma Working Group. Criteria for the classification of monoclonal gammopathies, multiple myeloma and related disorders: a report of the International Myeloma Working Group. Br. J. Haematol. 121, 749-757 (2003).

19 Gao, X., Starmer, J. \& Martin, E. R. A multiple testing correction method for genetic association studies using correlated single nucleotide polymorphisms. Genet. Epidemiol. 32, 361-369 (2008).

20 Chen, B., Wilkening, S., Drechsel, M. \& Hemminki, K. SNP tools: A compact tool package for analysis and conversion of genotype data for MS-Excel. BMC Res. Notes 2 , 214 (2009).

21 Stephens, M. \& Scheet, P. Accounting for decay of linkage disequilibrium in haplotype inference and missing-data imputation. Am. J. Hum. Genet. 76, 449-462 (2005).

22 Stephens, M., Smith, N. J. \& Donnelly, P. A new statistical method for haplotype reconstruction from population data. Am. J. Hum. Genet. 68, 978-989 (2001).

23 Moore, J. H. Computational analysis of gene-gene interactions using multifactor dimensionality reduction. Expert Rev. Mol. Diagn. 4, 795-803 (2004).

Supplementary Information accompanies the paper on Journal of Human Genetics website (http://www.nature.com/jhg) 